\title{
Brain and Acute Leukemia, Cytoplasmic Gene Overexpression as a Prognostic Factor in Egyptian De novo Adult Acute Myeloid Leukemia Patients
}

\begin{abstract}
Background: Brain and acute leukemia, cytoplasmic (BAALC) gene is identified on chromosome $8 \mathrm{q} 22.3$ and implicated in normal hematopoiesis. BAALC gene overexpression is associated with poor outcome. Methods: We aimed to evaluate BAALC expression in de novo Egyptian acute myeloid leukemia (AML) cases and determine its prognostic value. We recruited 70 patients with de novo AML diagnosed and treated at clinical pathology and medical oncology departments, fulfilling inclusion criteria in our prospective study and evaluated BAALC expression level. Patients received induction therapy. The Institutional Review Board approved our study. Results: The mean age was 39.2 years \pm 11.87 , (18-60) with a male/female ratio of $3 / 2$. The cutoff value of BAALC as a prognostic factor was 2.11 with sensitivity $(86.1 \%)$, specificity $(80 \%)$, positive predictive value $(88.6 \%)$, and negative predictive value $(76.2 \%)(P<0.001), 43(61.4 \%)$ patients had high BAALC expression. Seventy-two percent of patients in the low BAALC group achieved complete remission (CR) compared to $42.1 \%$ in high BAALC expression group $(P=0.03)$. Patients with low BAALC $(123.1 \pm 4.9)$ had longer mean survival time than high BAALC group $(45.85 \pm 5.1)(P=0.000)$. Conclusion: High-BAALC expression is an adverse prognostic factor, with a higher risk of relapse, lower CR rates, and lower survival in Egyptian de novo AML patients.
\end{abstract}

Keywords: Acute myeloid leukemia, adult, brain and acute leukemia, cytoplasmic, de novo, prognostic

\section{Introduction}

Acute myeloid leukemia (AML) is a clonal myeloid neoplasm with myelopoiesis maturation arrest that leads to myeloblasts accumulation in the bone marrow (BM) and/or blood. According to the current WHO classification, myeloblasts must comprise at least $20 \%$ of nucleated cells in the $\mathrm{BM}$ or blood to establish a diagnosis of AML. ${ }^{[1]}$

Nowadays, therapeutic options for AML that incorporate therapies targeted at specific dysregulated genetic pathways are available. Therefore, 2008 WHO Classification of AML relies mainly on cytogenetic abnormalities and mutations in three oncogenes (NPM1, FLT3, and CEBPA) for genetic sub-classification. ${ }^{[1]}$

Deregulation expression of genes involved in cell survival, proliferation and differentiation, e.g., (brain and acute leukemia and cytoplasmic [BAALC]), ${ }^{[2,3]}$

\footnotetext{
This is an open access journal, and articles are distributed under the terms of the Creative Commons Attribution-NonCommercial-ShareAlike 4.0 License, which allows others to remix, tweak, and build upon the work non-commercially, as long as appropriate credit is given and the new creations are licensed under the identical terms.
}

For reprints contact: WKHLRPMedknow_reprints@wolterskluwer.com
ERG, MN1 and WT1, and EVI1 were identified as the prognostic markers. ${ }^{[4]}$

BAALC gene identified on chromosome $8 \mathrm{q} 22.3$ is implicated in normal hematopoiesis. However, BAALC function in the hematopoietic system and its contribution in leukemogenesis are not fully understood. BAALC blocks myeloid differentiation, thus requiring a second mutation to induce leukemogenesis. ${ }^{[5]}$

BAALC overexpression is an indicator of AML aggressiveness as it is associated with poor clinical outcome. ${ }^{[6]}$ High-BAALC expression is associated with lower CR rates, shorter event free survival, and shorter overall survival. ${ }^{[7]}$

\section{Methods}

\section{Study design}

We carried out this prospective cohort study in the Clinical Pathology Department, Scientific and Medical Research Center and Medical Oncology Department, Faculty of Medicine, from January 2015 and February

How to cite this article: Alnagar AA, Al Hagrassy HM, Abdullah RM, El Shabrawy RM, Salah H. Brain and acute leukemia, cytoplasmic gene overexpression as a prognostic factor in Egyptian de novo adult acute myeloid leukemia patients. Indian J Med Paediatr Oncol 2020;41:859-68.

\section{Ahmed A Alnagar', Hesham M Al Hagrassy ${ }^{2}$, Rania M Abdullah², Reham M El Shabrawy ${ }^{3}$, Hossam Salah ${ }^{2}$}

${ }^{1}$ Department of Medical Oncology, Faculty of Medicine, Zagazig University, Zagazig, Egypt, ${ }^{2}$ Department of Clinical Pathology, Faculty of Medicine, Zagazig University, Zagazig, Egypt, ${ }^{3}$ Department of Microbiology and Immunology, Faculty of Medicine, Zagazig University, Zagazig, Egypt

Submitted: 05-May-2020 Revised: 22-Aug-2020 Accepted: 02-Oct-2020 Published: 31-Dec-2020

\footnotetext{
Address for correspondence:

Dr. Ahmed A Alnagar,

Department of Medical

Oncology, Faculty of Medicine, Zagazig University,

Zagazig, Egypt.

E-mail:draalnagar@yahoo.com
}

Access this article online

Website: www.ijmpo.org

DOI: 10.4103/ijmpo.ijmpo_215_20 Quick Response Code:

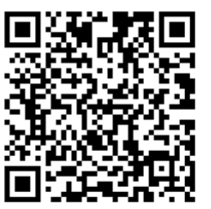


2019. The Faculty of Medicine Institutional Review Board and the Ethical Committee approved this study. We evaluated BAALC expression levels in acute myeloid leukemia patients and assessed its prognostic significance. A total of 70 patients were recruited, out of 107 patients in 12 months, informed consent was obtained from all patients. Furthermore, six individuals matched for age and sex were enrolled as internal control, patient's distribution is shown in Figure 1.

\section{Patient selection}

We enrolled in the study de novo adult AML patients aged $>18$ years old with normal liver, kidney and cardiac function tests with no concurrent malignancy and PS $\leq 2$. We excluded patients with secondary or relapsed AML or AML (M3).

Methods of brain and acute leukemia and cytoplasmic detection

We withdrew $2 \mathrm{ml}$ of venous blood aseptically from each patient by venipuncture into EDTA vacutainer for ESR. 2 $\mathrm{ml}$ of blood was directly used for RNA extraction.

Molecular detection of brain and acute leukemia and cytoplasmic RNA gene expression

RNA extraction from whole blood

We used Pure Link RNA Mini Kit (Life Technologies, USA) to purify RNA from anticoagulated BM sample.

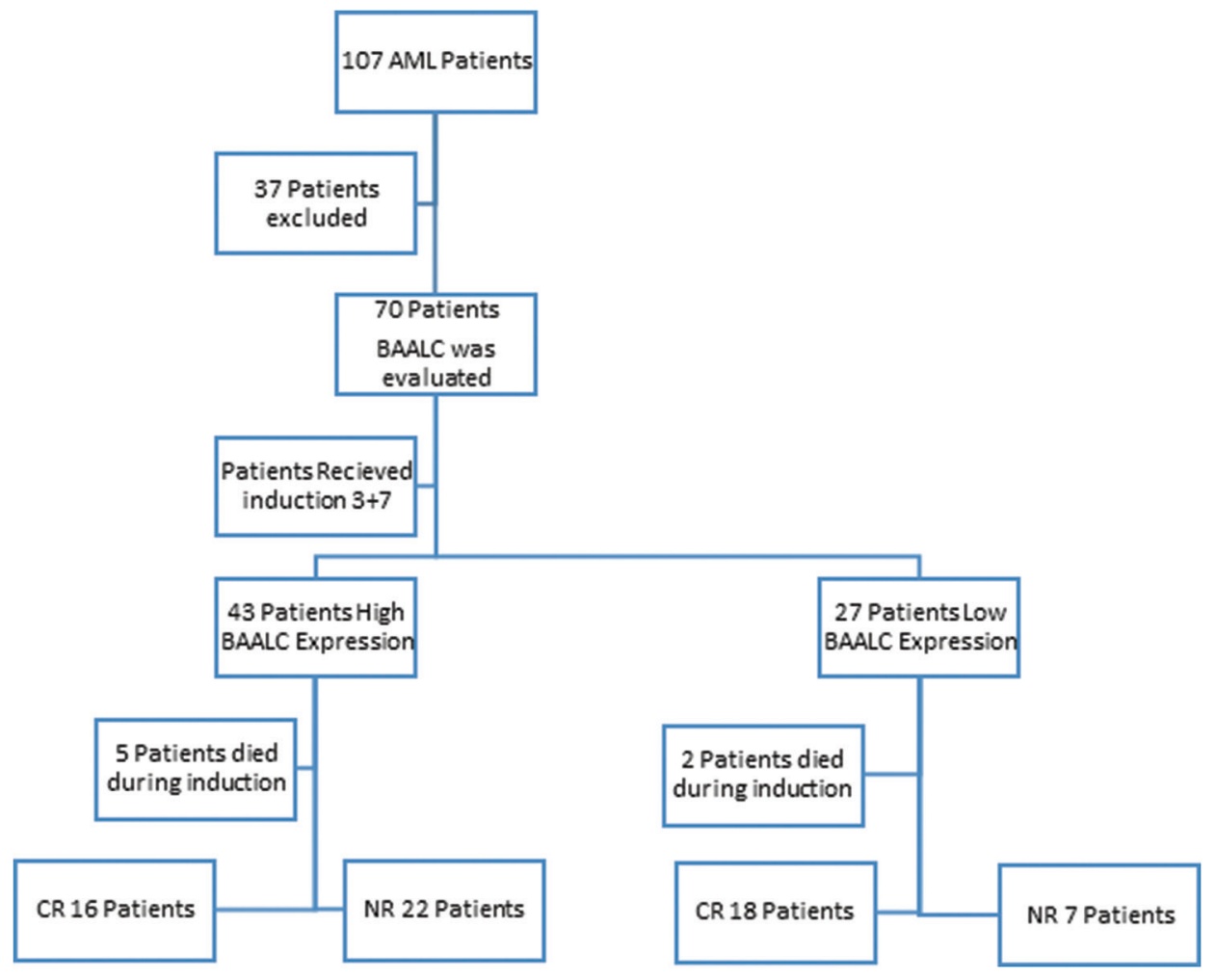

Reverse-transcription polymerase chain reaction of total RNA to cDNA

To synthesize single-stranded cDNA from total RNA, we use the high capacity cDNA reverse transcription kits:

We prepared the $2 \times$ Reverse Transcription Master Mix as per $20 \mu$ reaction.

Real-time reverse transcription polymerase chain reaction

Using Taqman gene expression assay.

Principle

Fluorescent dye intercalates into the amplification product, which enables the rapid analysis of target DNA during the polymerase chain reaction (PCR) process.

The real-time polymerase chain reaction

Using the stratagene $\mathrm{m} \times 3005 \mathrm{p}$ quantitative PCR system.

Interpretation of results

We normalized the transcription levels of target genes to those of B-actin (using a reference gene for accounting for the variability of cDNA amount in each sample).

Target genes expressed and presented as fold change of gene expression relative to controls. BAALC expression was positive if their expression level was one log higher than the mean level of expression reported for the control group, so the mean level for control was 1, samples were positive if expression level was more than $2 .{ }^{[8]}$ 


\section{Assessment}

We assessed patients demographics, clinical and laboratory data at the diagnosis (complete blood count [CBC], blood film, kidney function tests, serum uric acid, electrolytes level, liver function tests, coagulation profile, virology ( $\mathrm{HCV} \mathrm{Ab}, \mathrm{HBs} \mathrm{Ag}, \mathrm{HBc} \mathrm{Ab}$, and HIV Ab)), and CSF cytological analysis (if needed). Specific investigations include BMA, immunophenotyping, karyotyping, and reverse transcriptase PCR for BAALC gene expression. We treated the patients with induction regimen $(3+7$ protocol $)$ consisting of cytarabine $\left(100 \mathrm{mg} / \mathrm{m}^{2} / 24 \mathrm{~h}\right)$ continuous infusion daily for seven consecutive days, combined with doxorubicin $\left(30 \mathrm{mg} / \mathrm{m}^{2} / 24 \mathrm{~h}\right)$ for 3 days. An induction protocol consisting of $(2+14$ protocol) (cytarabine 10 $\mathrm{mg} / \mathrm{m}^{2} / 12 \mathrm{~h}$ ) daily for 14 days combined with 2 days of doxorubicin $\left(25 \mathrm{mg} / \mathrm{m}^{2}\right)$, was offered to patients who are over 60 years or to those who are between 55 and 60 with multiple comorbidities. We evaluated $\mathrm{CBC}$ and $\mathrm{BM}$ aspirate on day 14,28 to evaluate the remission state.

The patient achieved $\mathrm{CR}$ if $\mathrm{BM}$ was normocellular, containing $<5 \%$ blast cells and peripheral blood (PB) with at least $1 \times 109 / \mathrm{L}$ neutrophils and $\geq 100 \times 109 / \mathrm{L}$ platelets (PLTs). Remission failure included either partial remission (PR: Defined as $5 \%-15 \%$ blast cells), resistant disease (RD: Defined as $>15 \%$ blasts in BM), or induction death (ID: Defined as related to treatment or hypoplasia). We followed patients for 3 years to evaluate the survival.

Consolidation therapy: 3-4 cycles of high dose Ara-C $\left(1 \mathrm{~g} / \mathrm{m}^{2} / 12 \mathrm{~h}\right.$ at days $\left.1,3,5\right)$ postremission.

\section{Statistical analysis}

We used version 23.0. of IBM SPSS statistics (IBM Corp., Armonk, NY, USA); percentage analysis was done for categorical data; mean and standard deviation for continuous variables; survival was estimated using the Kaplan-Meier method. We evaluated the relationship between different prognostic and predictor variables with survival using the log-rank test. Independent prognostic variables affecting survival were evaluated using Cox-regression analysis. Tests were two-sided. $P \leq 0.05$ was considered as statistically significant, $P \leq 0.001$ as considered as highly significant.

\section{Results}

The present study included 70 patients. Forty-three patients $(61.4 \%)$ were males, 27 females, and their ages ranged from 19 to 58 years, with a mean of 39.2 years \pm 11.87 .

Patients' characteristics are described in Table 1, where 22 patients had cytogenetic abnormalities, 6 patients of M2 show $\mathrm{t}(8 ; 21), 7$ patients of M4 show inv (16), 3 patients of M1 and 2 patient from M5 show tri (8), and 4 patients of M5 show t $(11 ; 12)$.

\begin{tabular}{|c|c|}
\hline Variable & AML patients $(n=70), n(\%)$ \\
\hline Bone aches & $65(92.9)$ \\
\hline Fatigue & $57(81.4)$ \\
\hline Gum hypertrophy & $49(70)$ \\
\hline Purpura & $51(72.9)$ \\
\hline Bleeding & $50(71.4)$ \\
\hline Fever & $50(71.4)$ \\
\hline Splenomegaly & $11(15.7)$ \\
\hline Hepatomegaly & $11(15.7)$ \\
\hline Lymphadenopathy & $6(8.6)$ \\
\hline $\operatorname{TLC}\left(\times 10^{9} / \mathrm{L}\right)$, median (range) & $48.70(12-161)$ \\
\hline $\mathrm{Hb}(\mathrm{g} / \mathrm{dl})$, mean $\pm \mathrm{SD}$ (range) & $6.30 \pm 1.7(3.4-9.5)$ \\
\hline PLT $\left(\times 10^{9} / \mathrm{L}\right)$, median (range) & $28(5-100)$ \\
\hline PB blasts (\%), mean \pm SD (range) & $58.05 \pm 17.05(29-90)$ \\
\hline BM blasts (\%), mean $\pm \mathrm{SD}$ (range) & $74.23 \pm 13.18(52-95)$ \\
\hline $\operatorname{ESR}(\mathrm{mm} / \mathrm{h})$, mean $\pm \mathrm{SD}$ (range) & $111.1 \pm 17.16(90-150)$ \\
\hline LDH (IU/L), mean $\pm \mathrm{SD}$ (range) & $785.5 \pm 217.62(420-1200)$ \\
\hline \multicolumn{2}{|l|}{ FAB } \\
\hline With myeloid differentiation & $21(30)$ \\
\hline M1 & $3(4.3)$ \\
\hline M2 & $18(25.7)$ \\
\hline With monocytic differentiation & $49(70)$ \\
\hline M4 & $22(31.4)$ \\
\hline M5 & $27(38.6)$ \\
\hline \multicolumn{2}{|l|}{ Cytogenetics } \\
\hline Normal & $48(68.6)$ \\
\hline Abnormal & $22(31.4)$ \\
\hline \multicolumn{2}{|l|}{ Risk } \\
\hline Favorable & $13(18.6)$ \\
\hline$t(8 ; 21)$ & $6(8.6)$ \\
\hline inv (16) & $7(10)$ \\
\hline Intermediate & $53(75.7)$ \\
\hline Normal & $48(68.6)$ \\
\hline Tri 8 & $5(7.1)$ \\
\hline \multicolumn{2}{|l|}{ Adverse } \\
\hline$t(11 ; 12)$ & $4(5.7)$ \\
\hline
\end{tabular}

AML: Acute myeloid leukemia, TLC: Total leukocyte count, HB: Hemoglobin, PB: Peripheral blood, PLT: Platelet, BM: Bone marrow, ESR: Erythrocyte sedimentation rate, LDH: Lactate dehydrogenase, SD: Standard deviation, FAB: The FrenchAmerican-British classification of AML

Comparison between cytogenetic risk group AML patients as regard laboratory data showed that $\mathrm{Hb}, \mathrm{BM}$ blasts, lactate dehydrogenase (LDH), total leukocyte count (TLC), PLT and BAALC were the statistical difference between different cytogenetic risk groups, as shown in Table 2.

ROC curve for BAALC as a prognostic factor for survival in AML patients is shown in Figure 2 with sensitivity $(86.1 \%)$, specificity $(80 \%)$ with positive predictive value $(88.6 \%)$ and negative predictive value $(76.2 \%$.) with $P=0.000$ with cutoff value 2.11, where patients were classified into two groups based on BAALC value, also BAALC sensitivity 
Table 2: Comparison between cytogenetic risk and laboratory data, brain and acute leukemia, cytoplasmic among acute myeloid leukemia patients

\begin{tabular}{|c|c|c|c|c|}
\hline Variable & Normal cytogenetic $(n=48)$ & Favorable $(n=13)$ & Intermediate and adverse $(n=9)$ & $P$ \\
\hline Hb: Mean \pm SD & $5.69 \pm 1.21$ & $7.55 \pm 1.96$ & $7.00 \pm 2.53$ & $0.002 *$ \\
\hline BM blast: Mean \pm SD & $82.13 \pm 9.86$ & $61.91 \pm 9.49$ & $64.17 \pm 9.66$ & $0.000^{*}$ \\
\hline LDH: Mean \pm SD & $873.13 \pm 209.38$ & $634.55 \pm 115.63$ & $586.33 \pm 33$ & $0.000 *$ \\
\hline ESR: Mean \pm SD & $118.21 \pm 18.87$ & $127.55 \pm 9.53$ & $127.67 \pm 11.94$ & 0.172 \\
\hline TLC: Median (range) & $55(15-150)$ & $27(14-50)$ & $35(12-161)$ & $0.001^{*}$ \\
\hline PLT: Median (range) & $24(6-92)$ & $72(35-100)$ & $48.50(5-88)$ & $0.000^{*}$ \\
\hline PB blasts: Median (range) & $54(29-90)$ & $60(29-83)$ & $70(37-84)$ & 0.365 \\
\hline BAALC: Median (range) & $3.21(1.05-13.76)$ & $1.13(0.80-10.43)$ & $1.18(0.86-3.21)$ & $0.000^{*}$ \\
\hline
\end{tabular}

AML: Acute myeloid leukemia, TLC: Total leukocyte count, HB: Hemoglobin, PB: Peripheral blood, PLT: Platelet, BM: Bone marrow, ESR: Erythrocyte sedimentation rate, LDH: Lactate dehydrogenase, SD: Standard deviation, BAALC: Brain and Acute Leukemia, Cytoplasmic. *Statistically significant

Table 3: Sensitivity and specificity of brain and acute leukemia, cytoplasmic as a prognostic factor for survival

\begin{tabular}{|c|c|c|c|c|}
\hline \multicolumn{5}{|c|}{ Crosstab } \\
\hline \multirow{2}{*}{$\begin{array}{l}\text { BAALC } \\
\text { overexpression }\end{array}$} & \multirow[t]{2}{*}{ Variables } & \multicolumn{2}{|c|}{ Survival } & \multirow[t]{2}{*}{ Total } \\
\hline & & Die & Survive & \\
\hline \multicolumn{5}{|l|}{ BAALC2.11 } \\
\hline \multirow[t]{3}{*}{$>2.11$} & Count & 38 & 5 & 43 \\
\hline & $\begin{array}{l}\text { Percentage within } \\
\text { BAALC } 2.11\end{array}$ & 84.4 & 11.6 & 100.0 \\
\hline & Percentage within survival & 86.1 & 20.0 & 62.5 \\
\hline \multirow[t]{3}{*}{$\leq 2.11$} & Count & 7 & 20 & 27 \\
\hline & $\begin{array}{l}\text { Percentage within } \\
\text { BAALC } 2.11\end{array}$ & 25.9 & 74.1 & 100.0 \\
\hline & Percentage within survival & 15.6 & 80.0 & 37.5 \\
\hline \multirow[t]{3}{*}{ Total } & Count & 45 & 25 & 70 \\
\hline & $\begin{array}{l}\text { Percentage within } \\
\text { BAALC2.11 }\end{array}$ & 64.3 & 35.7 & 100.0 \\
\hline & Percentage within Survival & 100.0 & 100.0 & 100.0 \\
\hline
\end{tabular}

BAALC: Brain and acute leukemia, cytoplasmic

and specificity as a prognostic factor for survival is shown in Table 3.

BAALC high and low groups were compared using the MannWhitney $U$ test regard age, laboratory and clinical data and response to therapy; our results showed $\mathrm{HB}, \mathrm{BM}$ blast, $\mathrm{LDH}$, TLC, PLT, gum hypertrophy, bleeding, purpura, cytogenetics, and response to induction therapy differ significantly between BAALC high and low groups, as shown in Table 4.

\section{Treatment}

Different responses to induction therapy between both BAALC high or low is shown in Table 4, 66 AML patients received induction chemotherapy " $3+7$ " while 4 received $2+14$ protocol. BMA was used to assess response at day 14, it was done for 65 patients (5 patients had induction death). At D28, the response was assessed using BMA, 63 patients were evaluated, 2 patients lost after D14 evaluation (induction death).

Factors that affected survival significantly on univariate analysis were entered for multivariate analysis in

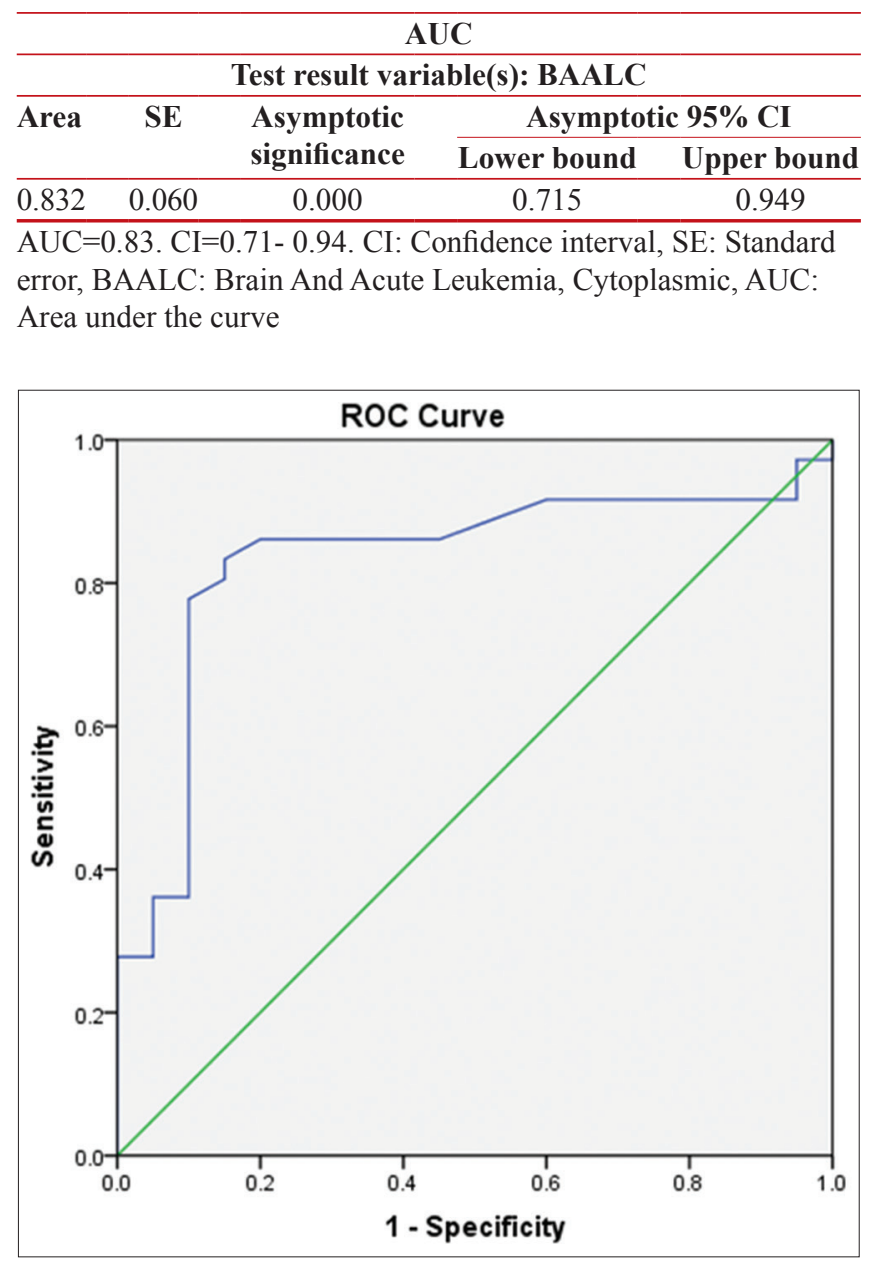

Figure 2: Receptor operating characteristic curve for brain and acute leukemia, cytoplasmic as a prognostic factor for survival in acute myeloid leukemia patients

cox-proportional hazard model. There was a statistically significant association between survival and gum hypertrophy, i.e., patients with gum hypertrophy are at a risk of dying 2.2 more than those with no gum hypertrophy, cytogenetics, i.e., patients with abnormal cytogenetics carry less risk 0.45 to die than those with normal cytogenetics, laboratory data, i.e., patients with low hemoglobin level, 
increased BM blasts, increased LDH level, higher TLC, lower PLT count, increased PB blasts, and high BAALC expression are at risk to die [Table 5].

When we evaluated mean overall survival time according to chemotherapy response, cytogenetics and BAALC among studied AML patients by Kaplan-Meier test, we found that mean survival time for patients who achieve complete remission (CR) $(121.1 \pm 4.9)$ was statistically significantly longer than those who not achieve CR (34.2 \pm 2.8$)$ (log-rank test $=62.62, P=0.000)$. Mean survival time for cytogenetically normal AML patients $(60.25 \pm 7.13)$ was shorter than favorable group $(114.00 \pm 8.64)$ and (patients with abnormal cytogenetics in intermediate and poor-risk groups $(107.16 \pm 16.71)(\log$-rank test $=10.83$, $P=0.004)$, also patients with low BAALC (123.1 \pm 4.9$)$ had longer mean survival time than patients with high BAALC $(45.85 \pm 5.1)$ (log-rank test $=31.20, P=0.000)$ as shown in Table 6 and Figure 3.

Forty-five patient died 45/70 (64.3\%). 3 (4.3\%) patients died initially before starting induction therapy related to disease (early death), 4 (5.7\%) patients died throughout induction chemotherapy period (induction death), 7 (10\%) died during consolidation due to chemotherapy toxicity, and $29(41.4 \%)$ patients died as a result of salvage chemotherapy toxicity in nonresponding patients, cause of death was mainly toxicity of chemotherapy (septic shock, heart failure, acute respiratory distress syndrome, and cardiogenic shock), in our study causes of early deaths in our study were related to disease progression (i.e., septic shock, DIC, cerebral haemorrhage and multiorgan failure).

Concerning toxicity; nearly all patients developed variable degrees of haematological toxicities, there was no difference regard toxicity pattern between both BAALC high and low group, although sepsis was numerically more common during induction in BAALC high group. Toxicity was listed in Table 7.

\section{Discussion}

In this study, we analyzed BAALC gene expression in 58 adult de novo AML patients (18-60 years) and evaluated its prognostic significance.

Typically, BAALC mRNA isn't detected in normal individuals; there was a statistically significant difference in expression between patients group and control, similar to what was reported by other studies..$^{[2,9]}$

We found no correlation between age and BAALC gene expression, that agreed with what reported in other studies by Bienz et al., ${ }^{[10]}$ and Metzeler et $a l .{ }^{[4]}$ That differs from what was stated by Rashed et al., ${ }^{[1]}$ who detected a significant correlation between high BAALC gene expression and age. Patients older than 45 years had higher
High BAALC expression. To confirm such an association, larger sample size may be needed.

BAALC gene expression no significant association different between sex, similar to what was declared in other studies. ${ }^{[7,11,12]}$

Patients in normal cytogenetic risk group had higher BAALC overexpression than the other two groups; this concurs with Zhou et al. ${ }^{[13]}$

We found that patients with CN-AML had higher (TLC, LDH level and blast cell count) and lower (PLT count and $\mathrm{Hb}$ level) that were statistically significant compared to the other groups. In patients with CN-AML median BAALC was 3.21 , that was a statistically significant difference than the other two groups. This agreed with Zhou et al., ${ }^{[13]}$ where BAALC median was 3.74.

Our cut off value for BAALC gene expression was $>2.11$ with sensitivity $86.1 \%$ and specificity $80 \%$, near to what stated by Zhou et al., where the cut-off value was $>2.35$ with sensitivity 55\% and specificity 100 .

No significant difference between both BAALC high expressed and low expressed patients regard age distribution, this agrees with Soliman et al., ${ }^{[14]}$ in contrary to Zhou et al., ${ }^{[13]}$ Rashed et al., ${ }^{[1]}$ who recorded that low BAALC expresser patients were significantly younger than high BAALC expresser patients.

Patients with low BAALC expression had significant lower BM blasts than in high BAALC expression group that work in with Qi et al., ${ }^{[15]}$ Zhou et al., ${ }^{[13]}$ Schwind et al. did not confirm these findings, ${ }^{[16]}$ and Weber et al., ${ }^{[7]}$ who did not found the difference in BM blasts between low BAALC expressed and high BAALC expressed groups. We could be owed this to the differences in the distribution of AML subtype or ethnicity.

Regard correlation between BAALC expression and response rate was found that patient with low BAALC expression achieved higher CR rate $(70 \%)$ in comparison to patients with high BAALC (40\%), this agrees with El-Sharnouby et al., ${ }^{[17]}$ and Yahya et al., ${ }^{[18]}$ who found that frequency of $\mathrm{CR}$ was higher in low BAALC expresser patients than in high expressers $71.4 \%$ versus $50 \%$ and $73.9 \%$ versus $22.7 \%$ respectively. Also, patients with high BAALC expression had a significantly worse clinical outcome than low expressers.

Regard survival, we found low BAALC expression patients had significantly longer overall survival than high BAALC expression patients (123 vs. 45 weeks respectively), and this came in concordance with Zhou et al., ${ }^{[13]}$ and Yahya et al., ${ }^{[18]}$ who shows superior overall survival (15.02 vs. 7.02 months) in low BAALC expressers.

In concordance with what stated by Bienz et al., ${ }^{[10]}$ Baldus et al., ${ }^{[2]}$ Langer et al., ${ }^{[3]}$ Langer et al., 2009 ${ }^{[19]}$; 


\begin{tabular}{|c|c|c|c|c|}
\hline \multirow[t]{2}{*}{ Variable } & \multicolumn{2}{|c|}{ BAALC } & \multirow[t]{2}{*}{ Test } & \multirow[t]{2}{*}{$P$} \\
\hline & $>2.11(n=43)$ & $\leq 2.11(n=27)$ & & \\
\hline Age: Mean \pm SD & $38.03 \pm 12.76$ & $42.24 \pm 9.96$ & $t=1.29$ & 0.202 \\
\hline $\mathrm{Hb}: \mathrm{Mean} \pm \mathrm{SD}$ & $5.51 \pm 1.26$ & $7.33 \pm 1.77$ & $t=4.46$ & $0.000 *$ \\
\hline BM blasts: Mean \pm SD & $85.20 \pm 6.09$ & $61.29 \pm 6.27$ & $t=14.06$ & $0.000^{*}$ \\
\hline LDH: Mean \pm SD & $924.74 \pm 164.47$ & $580.19 \pm 79.66$ & $t=8.96$ & $0.000^{*}$ \\
\hline ESR: Mean \pm SD & $121.72 \pm 17.26$ & $119.85 \pm 35$ & $t=0.38$ & 0.699 \\
\hline TLC: Median (range) & $60(33-161)$ & $29(12-52)$ & $\mathrm{MW}=4.68$ & $0.000^{*}$ \\
\hline Plt: Median (range) & $20(5-40)$ & $60(30-100)$ & $\mathrm{MW}=5.90$ & $0.000^{*}$ \\
\hline PB blasts: Median (range) & $58(29-90)$ & $60(29-84)$ & $\mathrm{MW}=0.24$ & 0.806 \\
\hline \multicolumn{5}{|l|}{ Sex, $n(\%)$} \\
\hline Female (27) & $17(63)$ & $10(37)$ & $\chi^{2}=0.02$ & 0.888 \\
\hline Male (43) & $26(60.5)$ & $17(39.5)$ & & \\
\hline \multicolumn{5}{|l|}{ Fatigue, $n(\%)$} \\
\hline Yes (57) & 35 (61.4) & $22(38.6)$ & Fisher exact test & 1 \\
\hline No (13) & $8(61.5)$ & $5(38.5)$ & & \\
\hline \multicolumn{5}{|l|}{ Fever, $n(\%)$} \\
\hline Yes (50) & $31(62)$ & $19(38)$ & $\chi^{2}=0.141$ & 0.708 \\
\hline No (20) & $12(60)$ & $8(40)$ & & \\
\hline \multicolumn{5}{|l|}{ Bone ache, $n(\%)$} \\
\hline Yes (64) & $40(64.5)$ & $24(37.5)$ & $\chi^{2}=0.287$ & 0.592 \\
\hline No (6) & $3(50)$ & $3(50)$ & & \\
\hline \multicolumn{5}{|l|}{ Gum hypertrophy, $n(\%)$} \\
\hline Yes (49) & $41(83.5)$ & $8(16.5)$ & $\chi^{2}=27.25$ & $0.000 *$ \\
\hline No (21) & $2(6.7)$ & $19(93.3)$ & & \\
\hline \multicolumn{5}{|l|}{ Bleeding, $n(\%)$} \\
\hline Yes $(50)$ & $36(72)$ & $14(28)$ & $\chi^{2}=5.97$ & $0.015^{*}$ \\
\hline No (20) & $7(37.5)$ & $13(62.5)$ & & \\
\hline \multicolumn{5}{|l|}{ Purpura, $n(\%)$} \\
\hline Yes $(51)$ & $36(70.6)$ & $15(29.4)$ & $\chi^{2}=7.4$ & $0.006^{*}$ \\
\hline No (19) & $7(36.8)$ & $12(63.2)$ & & \\
\hline \multicolumn{5}{|l|}{ Splenomegaly, $n(\%)$} \\
\hline Yes (11) & $7(63.6)$ & $4(36.4)$ & $\chi^{2}=0.07$ & 0.778 \\
\hline No (59) & $36(61)$ & $23(39)$ & & \\
\hline \multicolumn{5}{|l|}{ Hepatomegaly, $n$ (\%) } \\
\hline Yes (11) & $7(63.6)$ & $4(36.4)$ & $\chi^{2}=0.07$ & 0.778 \\
\hline No (59) & $36(61)$ & $23(39)$ & & \\
\hline \multicolumn{5}{|l|}{ LN, $n(\%)$} \\
\hline Yes (6) & $4(66.7)$ & $2(33.3)$ & $\chi^{2}=0.71$ & 0.397 \\
\hline No (64) & $39(60.9)$ & $25(39.1)$ & & \\
\hline \multicolumn{5}{|l|}{ Cytogenetics, $n(\%)$} \\
\hline Abnormal (22) & $3(13.6)$ & $19(86.4)$ & $\chi^{2}=26.80$ & $0.000^{*}$ \\
\hline Normal (48) & $40(83.3)$ & $8(16.7)$ & & \\
\hline Induction protocol (70), $n(\%)$ & & & & 0.33 \\
\hline $3+7(66[94.3 \%])$ & $39(90.7)$ & $27(100)$ & & \\
\hline $2+14(4[5.7 \%])$ & $4(9.3)$ & $0(0)$ & & \\
\hline Total $70(100 \%)$ & $43(100)$ & $27(100)$ & & \\
\hline$B M D 14$ & & & & 0.22 \\
\hline \multicolumn{5}{|l|}{ Evaluated patients (65[92.9\%]) } \\
\hline Unevaluated patients $(5[7.1 \%])$ & & & & \\
\hline Blasts \% (median, range) $(0-40)$ & $1.5(0-12)$ & $5(0-40)$ & & \\
\hline
\end{tabular}




\begin{tabular}{|c|c|c|c|c|}
\hline \multicolumn{5}{|c|}{ Table 4: Contd... } \\
\hline \multirow[t]{2}{*}{ Variable } & \multicolumn{2}{|c|}{ BAALC } & \multirow[t]{2}{*}{ Test } & \multirow[t]{2}{*}{$P$} \\
\hline & $>2.11(n=43)$ & $\leq 2.11(n=27)$ & & \\
\hline \multicolumn{5}{|l|}{ BM D28 } \\
\hline \multicolumn{5}{|l|}{ Evaluated patients [63 (90\%)] } \\
\hline \multicolumn{5}{|l|}{ Unevaluated (7 [10\%]) } \\
\hline \multicolumn{3}{|l|}{ Cellularity, $n(\%)$} & & \multirow[t]{4}{*}{0.88} \\
\hline Normocellular (39[62\%]) & $24(63.1)$ & $15(60)$ & & \\
\hline Hypocellular (12 [19\%]) & $7(18.4)$ & $5(20)$ & & \\
\hline Hypercellular (12 [19\%]) & $7(18.4)$ & $5(20)$ & & \\
\hline Total & $38(100)$ & $25(100)$ & & 0.07 \\
\hline Blasts $\%$ (median, range) $2(0-21)$ & $2(0-21)$ & $3(1-20)$ & & \\
\hline \multicolumn{5}{|l|}{ Induction response $(n=63), n(\%)$} \\
\hline CR $(34[54 \%])$ & $16(42.1)$ & $18(72)$ & & \multirow[t]{3}{*}{0.03} \\
\hline Not in CR (29 [46\%]) & $22(57.9)$ & $7(28)$ & & \\
\hline Total & $38(100)$ & $25(100)$ & & \\
\hline \multicolumn{5}{|c|}{$\begin{array}{l}\text { ANOVA, } t \text { : T-test, MW: Mann- Whitney-U test. AML: Acute myeloid leukemia, TLC: Total leukocyte count, HB: Hemoglobin, PB: } \\
\text { Peripheral blood, PLT: Platelet, BM: Bone marrow, ESR: Erythrocyte sedimentation rate, LDH: Lactate dehydrogenase, SD: Standard } \\
\text { deviation, BAALC: Brain and Acute Leukemia, Cytoplasmic, LN: Lymphadenopathy }\end{array}$} \\
\hline \multicolumn{5}{|c|}{$\begin{array}{c}\text { Table 5: Association between survival and demographic, clinical and laboratory data among studied acute myeloid } \\
\text { leukemia patients }\end{array}$} \\
\hline \multirow[t]{2}{*}{ Variable } & \multicolumn{2}{|c|}{ Survival } & \multirow{2}{*}{\multicolumn{2}{|c|}{$P \pm R R(95 \%$ CI) }} \\
\hline & Die (45) & Survive (25) & & \\
\hline \multicolumn{5}{|l|}{ Sex, $n(\%)$} \\
\hline Female (27) & $19(70.4)$ & $8(29.6)$ & \multirow{2}{*}{\multicolumn{2}{|c|}{0.625 RR $1.1(0.74-1.62)$}} \\
\hline Male (43) & $26(60.5)$ & $17(39.5)$ & & \\
\hline \multicolumn{5}{|l|}{ Fatigue, $n(\%)$} \\
\hline Yes (57) & $36(63.2)$ & $21(36.8)$ & \multirow{2}{*}{\multicolumn{2}{|c|}{0.96 RR $1.0(0.61-1.66)$}} \\
\hline No (13) & $9(69)$ & $4(31)$ & & \\
\hline \multicolumn{5}{|l|}{ Fever, $n(\%)$} \\
\hline Yes $(50)$ & $32(64)$ & $18(36)$ & $0.96 \mathrm{RR}$ & \\
\hline No (20) & $13(65)$ & $7(35)$ & & \\
\hline Bone ache, $n(\%)$ & & & & \\
\hline Yes (64) & $42(65.6)$ & $22(34.4)$ & $0.53 \mathrm{RR}$ & \\
\hline No (6) & $3(50)$ & $3(50)$ & & \\
\hline Gum hypertrophy, $n(\%)$ & & & & \\
\hline Yes (49) & $37(75.5)$ & $12(24.5)$ & $0.003 * \mathrm{R}$ & \\
\hline No (21) & $8(38)$ & $13(62)$ & & \\
\hline Bleeding, $n(\%)$ & & & & \\
\hline Yes $(50)$ & $33(66)$ & $17(34)$ & $0.86 \mathrm{RR}$ & \\
\hline No (20) & $12(60)$ & $8(40)$ & & \\
\hline Purpura, $n(\%)$ & & & & \\
\hline Yes $(51)$ & $33(64.8)$ & $18(35.2)$ & $0.68 \mathrm{RR}$ & \\
\hline No (19) & $12(63.2)$ & $7(36.8)$ & & \\
\hline Splenomegaly, $n(\%)$ & & & & \\
\hline Yes (11) & $7(63.6)$ & $4(36.4)$ & $0.87 \mathrm{RR}$ & \\
\hline No (59) & $38(64.4)$ & $21(35.6)$ & & \\
\hline Hepatomegaly, $n(\%)$ & & & & \\
\hline Yes (11) & $7(63.6)$ & $4(36.4)$ & $0.87 \mathrm{RR}$ & \\
\hline No (59) & $38(64.4)$ & $21(35.6)$ & & \\
\hline $\mathrm{LN}, n(\%)$ & & & & \\
\hline Yes (6) & $4(66.7)$ & $2(33.3)$ & $0.44 \mathrm{RR}$ & \\
\hline No (64) & $41(64.1)$ & $23(35.9)$ & & \\
\hline
\end{tabular}




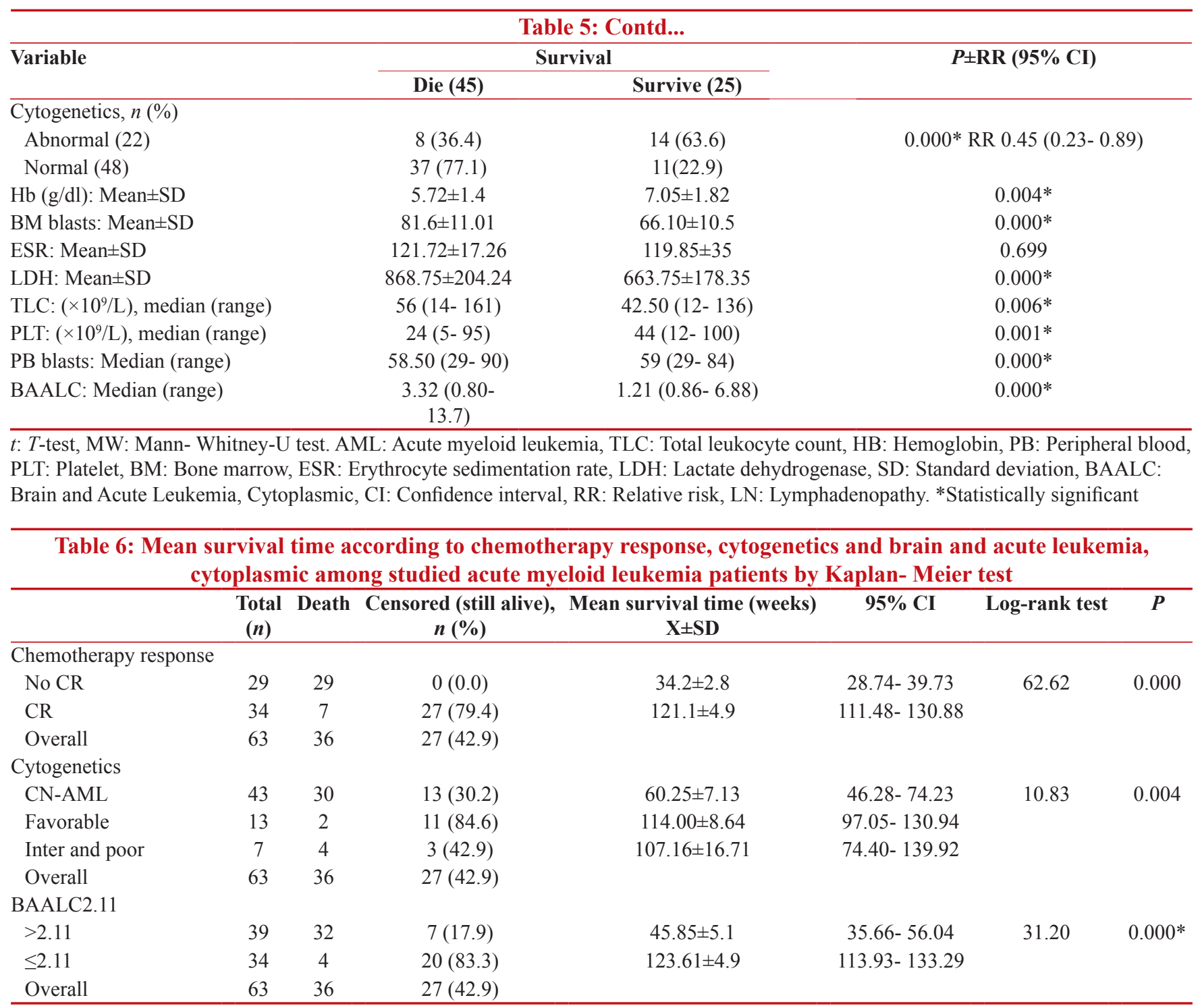

CI: Confidence interval, BAALC: Brain and Acute Leukemia, Cytoplasmic, AML: Acute myeloid leukemia, SD: Standard deviation

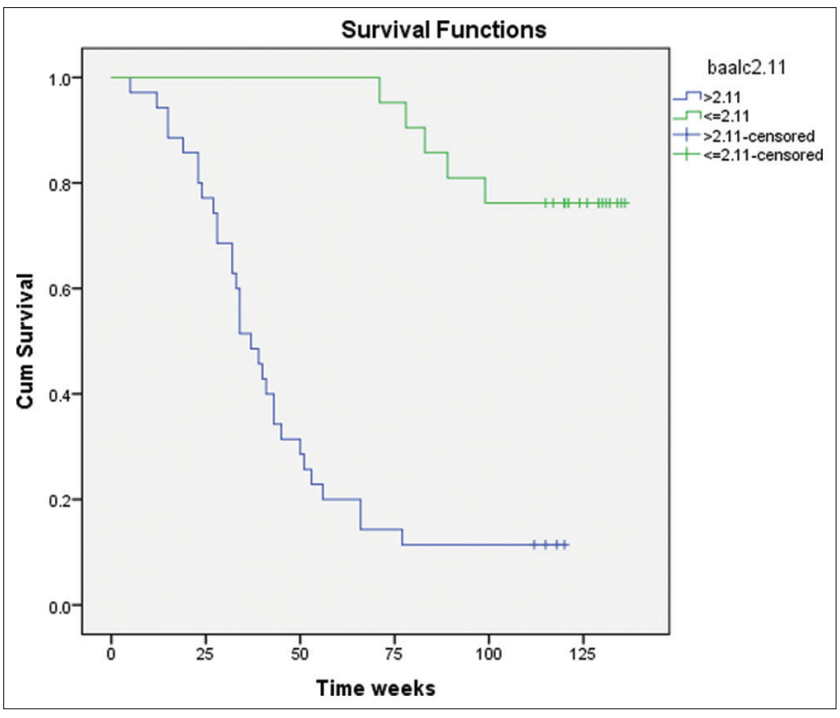

Figure 3: Cumulative survival time according to brain and acute leukemia, cytoplasmic among studied acute myeloid leukemia patients by KaplanMeier test
Schwind et al., ${ }^{[16]}$ and Eisfeld et al., ${ }^{[20]}$ we found that The likely hood of death was almost twice in high BAALC expression patients compared to low BAALC expression patients.

\section{Limitation of our study}

Risk stratification based on only cytogenetics. Were molecular markers such as FLT3, IDH1, NPM1. We used Doxorubicine as it is the available anthracycline in our institute.

\section{Conclusion}

AML patients had increased BAALC gene expression. High BAALC expression is associated with a higher risk of relapse and lower rates of CR. This molecular marker is specific significant associated with response to treatment, disease progression and survival. So its overexpression is a poor prognostic marker, it might be of a potential target for the evolution of new therapies. 


\begin{tabular}{|c|c|c|}
\hline Toxicity type & $\begin{array}{c}\text { During } \\
\text { induction } \\
(n=63), n(\%)\end{array}$ & $\begin{array}{c}\text { During } \\
\text { consolidation } \\
(n=56), n(\%)\end{array}$ \\
\hline \multicolumn{3}{|c|}{ Hematological toxicity } \\
\hline \multicolumn{3}{|l|}{ Neutropenia } \\
\hline G3 & $0(0)$ & $4(7)$ \\
\hline G4 & $63(100)$ & $52(93)$ \\
\hline \multicolumn{3}{|l|}{ Anemia } \\
\hline G3 & $53(84.1)$ & $45(80)$ \\
\hline G4 & $10(15.9)$ & $11(20)$ \\
\hline \multicolumn{3}{|c|}{ Thrombocytopenia } \\
\hline G3 & $0(0)$ & $3(5.4)$ \\
\hline G4 & $63(100)$ & $53(94.6)$ \\
\hline \multicolumn{3}{|c|}{ Fever and infection } \\
\hline \multicolumn{3}{|c|}{ Neutropenic fever } \\
\hline G3 & $55(87.3)$ & $45(80)$ \\
\hline G4 & $8(12.7)$ & $11(20)$ \\
\hline \multicolumn{3}{|c|}{ Cannula site infection } \\
\hline G2 & $0(0)$ & $4(7)$ \\
\hline G3 & $3(4.8)$ & $3(5.4)$ \\
\hline G4 & $1(1.6)$ & $1(1.8)$ \\
\hline \multicolumn{3}{|l|}{ Sepsis } \\
\hline G3 & $1(1.6)$ & $4(7)$ \\
\hline G4 & $7(11.1)$ & $1(1.8)$ \\
\hline \multicolumn{3}{|c|}{ Chest infection } \\
\hline G3 & $9(14.3)$ & $12(21.4)$ \\
\hline G4 & $3(4.8)$ & $0(0)$ \\
\hline \multicolumn{3}{|c|}{ Soft-tissue infection } \\
\hline G3 & $1(1.6)$ & $2(3.6)$ \\
\hline G4 & $2(3.2)$ & $1(1.8)$ \\
\hline \multicolumn{3}{|l|}{ Typhlitis } \\
\hline G3 & $4(6.3)$ & $2(3.6)$ \\
\hline G4 & $2(3.2)$ & $1(1.8)$ \\
\hline \multicolumn{3}{|c|}{ Fungal infection } \\
\hline G3 & $2(3.2)$ & $7(12.5)$ \\
\hline G4 & $6(9.5)$ & $4(7)$ \\
\hline \multicolumn{3}{|l|}{ Other toxicities } \\
\hline \multicolumn{3}{|l|}{ Gastritis } \\
\hline $\mathrm{G} 2$ & $15(23.8)$ & $8(14.3)$ \\
\hline G3 & 12 (19) & $12(21.4)$ \\
\hline \multicolumn{3}{|l|}{ Vomiting } \\
\hline $\mathrm{G} 2$ & $4(6.3)$ & $4(7)$ \\
\hline G3 & $2(3.2)$ & $2(3.6)$ \\
\hline \multicolumn{3}{|l|}{ Diarrhea } \\
\hline G2 & $3(4.8)$ & $4(7)$ \\
\hline G3 & $2(3.2)$ & $0(0)$ \\
\hline \multicolumn{3}{|c|}{$\begin{array}{l}\text { Liver enzymes } \\
\text { increased }\end{array}$} \\
\hline $\mathrm{G} 2$ & $2(3.2)$ & $5(8.9)$ \\
\hline G3 & $2(3.2)$ & $1(1.8)$ \\
\hline
\end{tabular}

Contd...

\begin{tabular}{lcc}
\hline & Table 7: Contd... & \\
\hline Toxicity type & $\begin{array}{c}\text { During } \\
\text { induction } \\
(\boldsymbol{n}=\mathbf{6 3}), \boldsymbol{n}(\%)\end{array}$ & $\begin{array}{c}\text { During } \\
\text { consolidation } \\
(\boldsymbol{n}=\mathbf{5 6}), \boldsymbol{n}(\%)\end{array}$ \\
\hline $\begin{array}{l}\text { Blood bilirubin } \\
\text { increased }\end{array}$ & \\
G2 & $2(3.2)$ & $2(3.6)$ \\
G3 & $3(4.8)$ & $0(0)$ \\
\hline According to CTCAE Version 5 0. CTCAE: Common
\end{tabular}

According to CTCAE Version 5.0. CTCAE: Common Terminology

Criteria for Adverse Events

\section{Acknowledgements}

We want to thank our patients for their participation and compliance.

\section{Financial support and sponsorship}

Nil.

\section{Conflicts of interest}

There are no conflicts of interest.

\section{References}

1. Vardiman JW, Arber DA, Brunning RD, Larson RA, Matutes E, Baumann I, et al., Therapy-related myeloid neoplasms. In: Swerdlow SH, Campo E, Harris NL, Jaffe ES, Pileri SA, Stein H, et al., editors. WHO Classification of Tumours of Haematopoietic and Lymphoid Tissues. Lyon: IARC; 2008. p. 127-9.

2. Baldus CD, Thiede C, Soucek S, Bloomfield CD, Thiel E, Ehninger G. BAALC expression and FLT3 internal tandem duplication mutations in acute myeloid leukemia patients with normal cytogenetics: Prognostic implications. J Clin Oncol 2006;24:790-7.

3. Langer C, Radmacher MD, Ruppert AS, Whitman SP, Paschka P, Mrózek $\mathrm{K}$, et al. High BAALC expression associates with other molecular prognostic markers, poor outcome, and a distinct gene-expression signature in cytogenetically normal patients younger than 60 years with acute myeloid leukemia: A cancer and leukemia Group B (CALGB) study. Blood 2008;111:5371-9.

4. Metzeler KH, Dufour A, Benthaus T, Hummel M, Sauerland MC, Heinecke A, et al. ERG expression is an independent prognostic factor and allows refined risk stratification in cytogenetically normal acute myeloid leukemia: A comprehensive analysis of ERG, MN1, and BAALC transcript levels using oligonucleotide microarrays. J Clin Oncol 2009;27:5031-8.

5. Heuser M, Berg T, Kuchenbauer F, Lai CK, Park G, Fung S, et al. Functional role of BAALC in leukemogenesis. Leukemia 2012;26:532-6.

6. Franzoni A, Passon N, Fabbro D, Tiribelli M, Damiani D, Damante G. Histone post-translational modifications associated to BAALC expression in leukemic cells. Biochem Biophys Res Commun 2012;417:721-5.

7. Weber S, Alpermann T, Dicker F, Jeromin S, Nadarajah N, Eder C, et al. BAALC expression: A suitable marker for prognostic risk stratification and detection of residual disease in cytogenetically normal acute myeloid leukemia. Blood Cancer J 2014;10:e173. 
8. Spanaki A, Perdikogianni C, Linardakis E, Kalmanti M. Quantitative assessment of PRAME expression in diagnosis of childhood acute leukemia. Leuk Res 2007;31:639-42.

9. Baldus CD, Tanner SM, Ruppert AS, Whitman SP, Archer KJ, Marcucci G, et al. BAALC expression predicts clinical outcome of de novo acute myeloid leukemia patients with normal cytogenetics: A cancer and leukemia Group B study. Blood 2003;102:1613-8.

10. Bienz M, Ludwig M, Leibundgut EO, Mueller BU, Ratschiller D, Solenthaler $\mathrm{M}$, et al. Risk assessment in patients with acute myeloid leukemia and a normal karyotype. Clin Cancer Res 2005;11:1416-24.

11. Rashed R, Kadry D, EL Taweel M, El Wahab NA, El Hameed TA. Relation of BAALC and ERG gene expression with overall survival in acute myeloid leukemia cases. Asian Pac J Cancer Prev 2015;16:7875-82.

12. Amirpour M, Ayatollahi H, Sheikhi M, Azarkerdar S, Shams SF. Evaluation of BAALC gene expression in normal cytogenetic acute myeloid leukemia patients in North-East of Iran. Med J Islam Repub Iran 2016;30:418.

13. Zhou JD, Yang L, Zhang YY, Yang J, Wen XM, Guo H, et al. Overexpression of BAALC: Clinical significance in Chinese de novo acute myeloid leukemia. Med Oncol 2015;32:386.

14. Soliman A, Aal AA, Afify R, Ibrahim N. BAALC and ERG expression in Egyptian patients with acute myeloid leukemia, relation to survival and response to treatment. Open Access Maced J Med Sci 2016;4:264-70.
15. Qi X, Shen Y, Cen J, Chen H, Sun Y, Sheng $\mathrm{H}$, et al Up-regulation of BAALC gene may be an important alteration in AML-M2 patients with $\mathrm{t}(8 ; 21)$ translocation. J Cell Mol Med 2008;12:2301-4.

16. Schwind S, Marcucci G, Maharry K, Radmacher MD, Mrózek K, Holland $\mathrm{KB}$, et al. BAALC and ERG expression levels are associated with outcome and distinct gene and microRNA expression profiles in older patients with de novo cytogenetically normal acute myeloid leukemia: A cancer and leukemia Group B study. Blood 2010;116:5660-9.

17. El-Sharnouby JA, Ahmed LM, Taha AM, Kamal O. Prognostic significance of CEBPA mutations and BAALC expression in acute myeloid leukemia Egyptian patients with normal karyotype. Egypt J Immunol 2008;15:131-43.

18. Yahya RS, Sofan MA, Abdelmasseih HM, Saudy N, Sharaf-Eldein MA. Prognostic implication of BAALC gene expression in adult acute myeloid leukemia. Clin Lab 2013;59:621-8.

19. Langer C, Marcucci G, Holland KB, Radmacher MD, Maharry K, Paschka P, et al. Prognostic importance of MN1 transcript levels, and biologic insights from MN1-associated gene and microRNA expression signatures in cytogenetically normal acute myeloid leukemia: A cancer and leukemia Group B study. J Clin Oncol 2009;27:3198-204.

20. Eisfeld AK, Marcucci G, Liyanarachchi S, Döhner K, Schwind S, Maharry $\mathrm{K}$, et al. Heritable polymorphism predisposes to high BAALC expression in acute myeloid leukemia. Proc Natl Acad Sci USA 2012;109:6668-73. 